\title{
An Integrated Bragg Grating Oxygen Sensor Using A Hydrophobic Sol-gel Layer Doped With An Organic Dye
}

\author{
D.J.Wales $^{1,2}$, R.M.Parker ${ }^{2}$, J.C.Gates ${ }^{2}$, M.C.Grossel ${ }^{1}$, P.G.R.Smith ${ }^{2}$ \\ 1. School of Chemistry, University of Southampton, Southampton, Hampshire, SO17 1BJ, U.K. \\ 2. Optoelectronics Research Centre, University of Southampton, Southampton, Hampshire, SO17 1BJ, U.K.
}

Oxygen sensing is required for the understanding of many chemical processes across a diverse set of fields including medicine, environmental science and chemical synthesis. Oxygen sensing can be achieved through the use of electronic sensors. However, there are limitations associated with electronic sensors including susceptibility to electromagnetic interference and presenting a spark risk in flammable environments. Optical fiber and integrated optical chemical sensors overcome these limitations of electrical based sensing methods.

It has been previously shown that a planar optical waveguide containing a Bragg grating is an effective method of detecting minute changes in refractive index [1]. The evanescent field of the optical mode within an unclad waveguide containing a Bragg grating interacts with a chemical analyte. This device works by detecting the associated effective index change when the analyte is altered. The change in effective index of the mode is directly proportional to the Bragg wavelength of the grating. Accurate spectral measurement provides high dynamic range with observable changes in the effective refractive index of $10^{-6}$.

The waveguides are fabricated in a flame hydrolysis deposited layer of germanium doped silica. Simultaneous definition of both a channel waveguide and Bragg gratings can be achieved via a process known as Direct Grating Writing. Reference [2] provides a thorough review on the fabrication and development of UV written planar Bragg grating sensors. This work looks at depositing a porous layer onto the surface of these sensors for oxygen detection, as shown in Figure 1 (a).

(a)

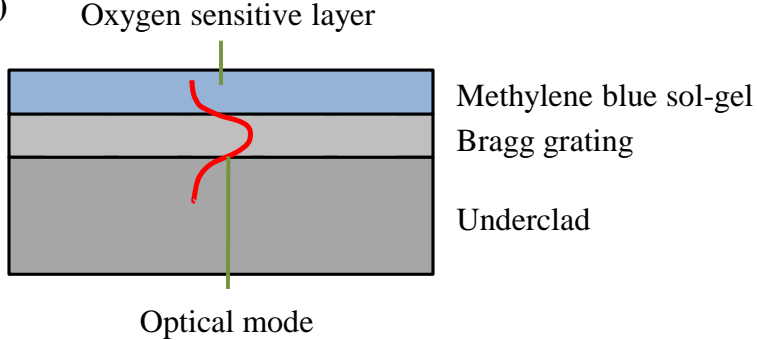

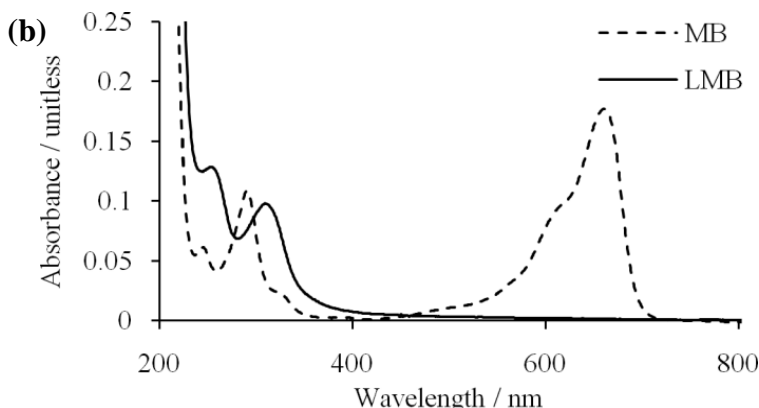

Wavelength / nm

Fig. 1 (a) Schematic of oxygen sensing device and (b) absorption spectra highlighting the colour change upon reduction of methylene blue (MB) to leuco-methylene blue (LMB).

Silica sol-gels are an inorganic porous matrix of a solid glass-like material [3]. Low temperature hydrolysis of tetraalkoxysilanes forms liquid silica sols which can be spin deposited and densified with heat treatment to form thin film layer/s. Silica sol-gel thin films have been widely utilised in sensing applications. However, silica solgels typically demonstrate cracking upon gelation and/or densification reducing the mechanical and optical qualities of the thin film. Non-cracking organically modified silica sol-gel thin films that were shown to encapsulate organic dyes have been demonstrated by Haruvy and Webber [4].

It is well known that methylene blue, an organic dye molecule, exhibits a reversible colour change upon exposure to oxygen [5] as shown in Figure 1(b). This quantitative change in absorption possesses an associated change in refractive index. We will show that methylene blue can be encapsulated within a porous, hydrophobic, non-cracking silica sol-gel. Spin deposition and heat treatment form a layer of the sol-gel several microns thick onto the integrated device to produce a gaseous oxygen sensor.

We shall present our latest work combining engineered oxygen sensitive sol-gels with high sensitivity integrated Bragg sensors for distributed oxygen sensing.

\section{References}

[1] R.M. Parker, J.C. Gates, M.C. Grossel, P.G.R. Smith, "In vacuo measurement of the sensitivity limit of planar Bragg grating sensors for monolayer detection ," App. Phys. Lett., 94, 173306 (2009)

[2] I.J.G. Sparrow, P.G.R. Smith, G.D. Emmerson, S.P. Watts, C. Riziotis, "Planar Bragg Grating Sensors - Fabrication and Applications: A Review," Journal of Sensors, 2009, 1-12 (2009)

[3] J.D. Wright \& N.A.J.M. Sommerdijk, Sol-gel Materials Chemistry and Applications (Taylor and Francis, London, 2001)

[4] Y. Haruvy \& S.E. Webber, "Supported Sol-Gel Thin-Film Glasses Embodying Laser Dyes. 1. A New Fast Method for the Preparation of Optically Clear Polysiloxane Thin-Film Glasses," Chem. Mater. 3, 501-507 (1991)

[5] J.A. Campbell, "Kinetics - Early and Often," J. Chem. Educ., 40, 578-583 (1963) 